Original Article

\title{
Principal motion analysis of manual stretching techniques for the ankle joints
}

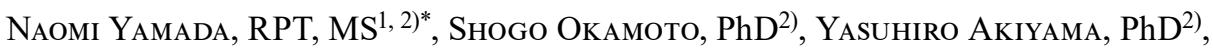 \\ YoJI YAMADA, $\mathrm{PhD}^{2)}$ \\ 1) Department of Rehabilitation, Aichi Medical College: 519 Ichiba, Kiyosu City, Aichi 452-0931, Japan \\ 2) Department of Mechanical Systems Engineering, Nagoya University, Japan
}

\begin{abstract}
Purpose] Physical therapists frequently perform manual stretching of the ankle joints. Manual stretching procedures are challenging to define because they involve multidirectional joint motions and external forces. Therefore, in this paper, we propose a method for quantitatively and statistically analyzing the manual foot stretching techniques used by physical therapists. [Participants and Methods] The participants were four physical therapists, and three patients who have a spastic foot. We investigated the manual foot stretching techniques employed by the physical therapists using a three-dimensional analysis system and an instrumented brace with force sensors. Principal motion analysis was applied to the obtained data, and principal motions were determined. [Results] The first principal motion was the application of force for the dorsiflexion of the foot; second, the pushing/pulling of the heel; third, the eversion/inversion of the entire foot; and fourth, the eversion/inversion of the forefoot. Furthermore, the manual stretching techniques varied among the physical therapists, even for the same patient, and some techniques occurred only between particular pairs. [Conclusion] This study demonstrated the effectiveness of the principal motion analysis for the statistical assessment of manual stretching techniques and clarifying differences in stretching technique among physical therapists. Key words: Principal motion analysis, Manual stretching, Ankle joint
\end{abstract}

(This article was submitted Apr. 24, 2020, and was accepted Jun. 22, 2020)

\section{INTRODUCTION}

Stretching is a frequently used treatment to increase the range of motion of the ankle joints, and several studies have proved its effectiveness ${ }^{1-4}$. Various studies have focused their investigations on the biomechanical changes in muscles and tendons due to stretching, using ultrasonography imaging devices, and have shown that muscle stiffness is decreased with stretching ${ }^{5-8)}$. In clinical practice, the therapist occasionally stretches the foot of the patient manually. While feeling the hardness of the target parts, the therapist adjusts the amount and direction of the applied force and performs manual stretching. However, no standard has been established for the degree of motion or force to be applied in foot stretching. Typically, stretching is performed on the basis of the knowledge and experience of individual therapists, which is the same for stretching procedures for all muscles. When multiple therapists treat the same patient, whether the procedure used by one therapist matches that used by another therapist is unknown.

Manual stretching is expressed as time-series data, with variables such as the three-dimensional force applied to the foot and the ankle joint angle. Such data can be analyzed using a principal motion analysis, which is an extension of the principal component analysis for time-series data; the method decomposes one motion into multiple independent motions, which are called principal motions. The number of principal motions is considerably smaller than the number of dimensions of the original motion; that is, a stretching motion is represented by the magnitudes of a few principal motions, which enables the

*Corresponding author. Naomi Yamada (E-mail: yamada-nao@yuai.ac.jp)

(C2020 The Society of Physical Therapy Science. Published by IPEC Inc.

(c) (1) $\odot$ This is an open-access article distributed under the terms of the Creative Commons Attribution Non-Commercial No DerivaCC BY NC ND tives (by-nc-nd) License. (CC-BY-NC-ND 4.0: https://creativecommons.org/licenses/by-nc-nd/4.0/) 
use of parametric testing for motion data and promotes statistical discussions. Park and Jo analyzed the motion of the upper limbs with multiple degrees of freedom by using the principal motion analysis ${ }^{9}$. With similar methods, Nakanishi et al. addressed walking and jumping motions ${ }^{10)}$, while Morishima et al. studied squat motions ${ }^{11)}$.

In this study, we applied the principal motion analysis to investigate manual stretching techniques for the ankle joints. The present study builds upon the study conducted by Yamada et al. ${ }^{12)}$ in which the force applied at the heel was analyzed. In the present study, the force applied at the heel and forefoot and the movements of the foot during stretching were simultaneously measured. Furthermore, we applied the principal motion analysis to the measured data and succeeded in extracting the features of the manual foot stretching technique in more detail. We also studied the manual stretching performed by multiple physical therapists for multiple patients to clarify the differences between the manual stretching techniques used by physical therapists. By employing patients, we investigated the adaptations of stretching techniques to the symptoms.

\section{PARTICIPANTS AND METHODS}

Four physical therapists, denoted as PT1, PT2, PT3, and PT4 (3 males and 1 female; mean age, $28.8 \pm 1.3$ years), with 5 to 8 years of experience in rehabilitation and three patients, denoted as ptA, ptB, and ptC ( 2 males and 1 female; mean age, 78.0 \pm 3.0 years), participated in the study. All the patients had spastic foot after stroke. Written informed consent was obtained from all the subjects. Each physical therapist performed manual stretching for each of the three patients. However, one physical therapist performed foot stretching on only 1 patient for business reasons. This study was approved by the Nagoya University Bioethics Review Committee and registered under approval No. 14-501.

An instrumented brace that can be attached to the foot of a patient was developed to measure the force applied by the physical therapist during manual foot stretching. Typically, while holding the heel of the patient, the physical therapist stretches the foot by pressing the forearm against the forefoot. To measure these contact forces, 2 six-axis force sensors were attached to the heel and the forefoot plantar surface of the brace (Fig. 1A). The patient lay on the bed, and the physical therapist stretched the spastic foot normally using the device. A three-dimensional analysis system (OptiTrack, NaturalPoint Inc., USA) was used to measure the foot posture. The force sensor and camera were synchronized and operated at $120 \mathrm{~Hz}$. For the anatomical positions, the $x$-axis was defined as the mediolateral axis; the $y$-axis, as the anteroposterior axis; and the $z$-axis, as the craniocaudal axis. The coordinates of the lower limb $\left(\Sigma_{l}\right)$ were determined from markers located on the tibia, and the coordinates of the foot $\left(\Sigma_{f}\right)$ were determined from the markers on the instrumented brace (Fig. 1B). The coordinates of the force sensor $\left(\Sigma_{s}\right)$ were set similarly. The rotation angle of $\Sigma_{f}$ on $\Sigma_{l}$ was defined as the foot posture $\left(\theta_{x}, \theta_{y}\right.$, and $\left.\theta_{z}\right)$ calculated with $x$-, $y$-, and $z$-axis rotations, in this order.

In one experiment, each physical therapist performed foot stretching for $10 \mathrm{~s}$ thrice at intervals of $5 \mathrm{~s}$ and performed three sets of such stretching. A rest period of approximately 1 min was provided between sets.

One stretch was divided into a transitional phase from the initial posture to dorsiflexion and a keeping phase, in which continuous stretching was performed in dorsiflexion. In this study, the transitional phase data were analyzed to confirm the posture change during stretching. The measurements from the starting to the end point were discretized into a data length of 100.

All data were normalized before the analysis. First, the differences between the maximum and minimum values of the joint angles, forces, and moments were calculated for the trial of each participant. Subsequently, each quantity was divided by the largest difference among all the trials.

The principal motion analysis was applied to the obtained time-series data, including 15 quantities. Foot posture is expressed as $\theta_{x}, \theta_{y}$, and $\theta_{z}$, and the triaxial force and moment applied to the heel are denoted by ${ }^{h} f_{x},{ }^{h} f_{y},{ }^{h} f_{z},{ }^{h} m_{x},{ }^{h} m_{y}$, and ${ }^{h} m_{z}$, and those for the forefoot are ${ }^{f} f_{x},{ }_{f},{ }_{f}, f_{m_{x}}, f_{m_{y}}$, and $f_{m_{z}}$. By performing the eigenvector expansion on the time-series data of all 90 trials, the principal motions and scores were calculated. The principal motions included typical motion patterns, and the score for each principal motion shows how much the feature of each principal motion was included in each trial. In this paper, four principal motions with large eigenvalues were adopted. These four movements can explain $87.6 \%$ of the variation in the measured data. For straightforward interpretation of each principal motion feature, Promax rotation was applied to the obtained results.

Two-way analysis of variance was applied to the scores of the first to the fourth principal motions obtained in the previous section to evaluate whether the manual stretching techniques used for multiple post-stroke patients differed among the physical therapists. For each principal motion, the main effects and interactions of the two factors, namely the physical therapist and the patient, were tested. One physical therapist (PT4) was excluded from the analysis because he performed stretching for only one patient. For the statistical analysis, MATLAB2016a was used.

\section{RESULTS}

Figure 2 shows the time transition of each variable from the first to the fourth principal motions. In the first principal motion, ${ }^{h} m_{x}$ and $f_{m_{x}}$ (the dorsiflexion moment applied to the heel and forefoot, respectively) significantly increased as the stretching phase progressed. Similarly, ${ }^{h} f_{y}$ (the force pushing the heel in the direction of the toe), ${ }^{f} f_{z}$ (the force pushing the forefoot in the direction of the dorsiflection), and $\theta_{x}$ (the dorsiflexion movement angle) also increased. From the above- 


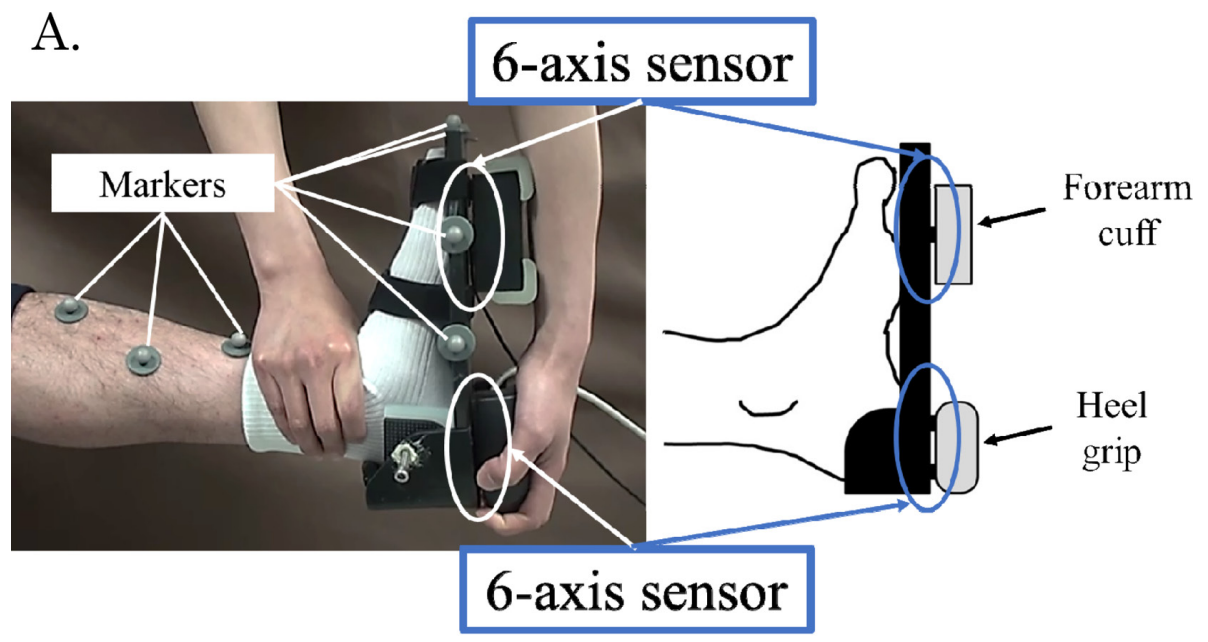

B.
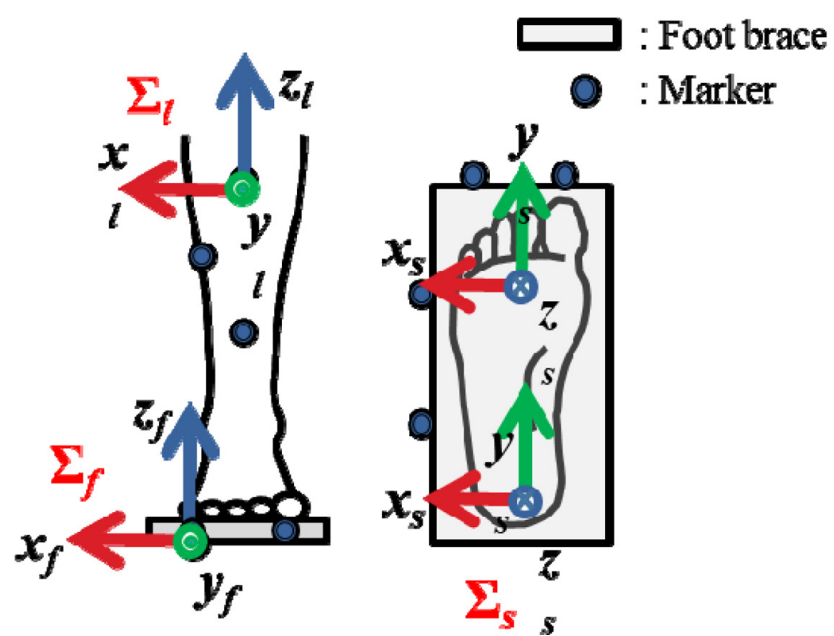

Fig. 1. A. Instrumented brace. Two six-axial force sensors are placed on the forefoot and heel areas of the brace. The physical therapist stretches the patient's foot while holding the heel grip and placing his/her forearm against the forearm cuff. B. Coordinate systems $\left(\Sigma_{l}, \Sigma_{f}\right.$, and $\left.\Sigma_{s}\right)$. l: lower limb, $f$ : foot, $s$ : sensor.

mentioned results, we judged that the first principal motion was to move the foot of the patient in the dorsiflexion direction. Typically, the first principal motion represents the average motion in all the trials.

In the second principal motion, only ${ }^{h} f_{z}$ (the force to push the heel toward the upper side) increased. This indicated that the second principal motion was a technique of pushing or pulling the heel.

In the third principal motion, although no significant temporal change occurred, $\theta_{y}$ (the inversion angle) was always positive, and ${ }^{f} f_{x}$ (the force pushing the forefoot outward) and $\theta_{x}$ (the dorsiflexion angle) decreased to negative values in the second half. Moreover, $f_{m_{y}}$ (the forefoot inversion moment) increased to a positive value in the latter phase. When these movements were combined, the entire foot was inverted. In other words, the third principal motion was a procedure used to turn the entire foot in or out.

In the fourth principal motion, $f_{m_{y}}$ (the inversion moment of the forefoot) greatly increased in the second half. Therefore, the fourth principal motion reflected whether the muscles involved in the inversion foot were consciously stretched.

Figure 3 shows a plot of the scores of the first to the fourth principal motions, with all trials being discerned for each physical therapist-patient combination. The scores of the first principal motion were positive in all the trials. The scores of the second principal motion tended to be negative for PT1 and PT4, and positive for PT2. In the third principal motion, the scores of PT4 were negative in all the trials. Furthermore, $\mathrm{ptC}$ tended to have negative scores. In the fourth principal motion, the scores of PT2 and PT4 tended to be negative. Moreover, the scores of PT1 were close to 0, while those of PT3 were widely distributed from positive to negative. Table 1 lists the mean and standard deviation of the scores of each patient-and-physical therapist pair and the results of the two-way analysis of variance of the scores in each trial for the first to the fourth principal motions. From the results, the differences in the techniques used by the physical therapists can be statistically discussed for 

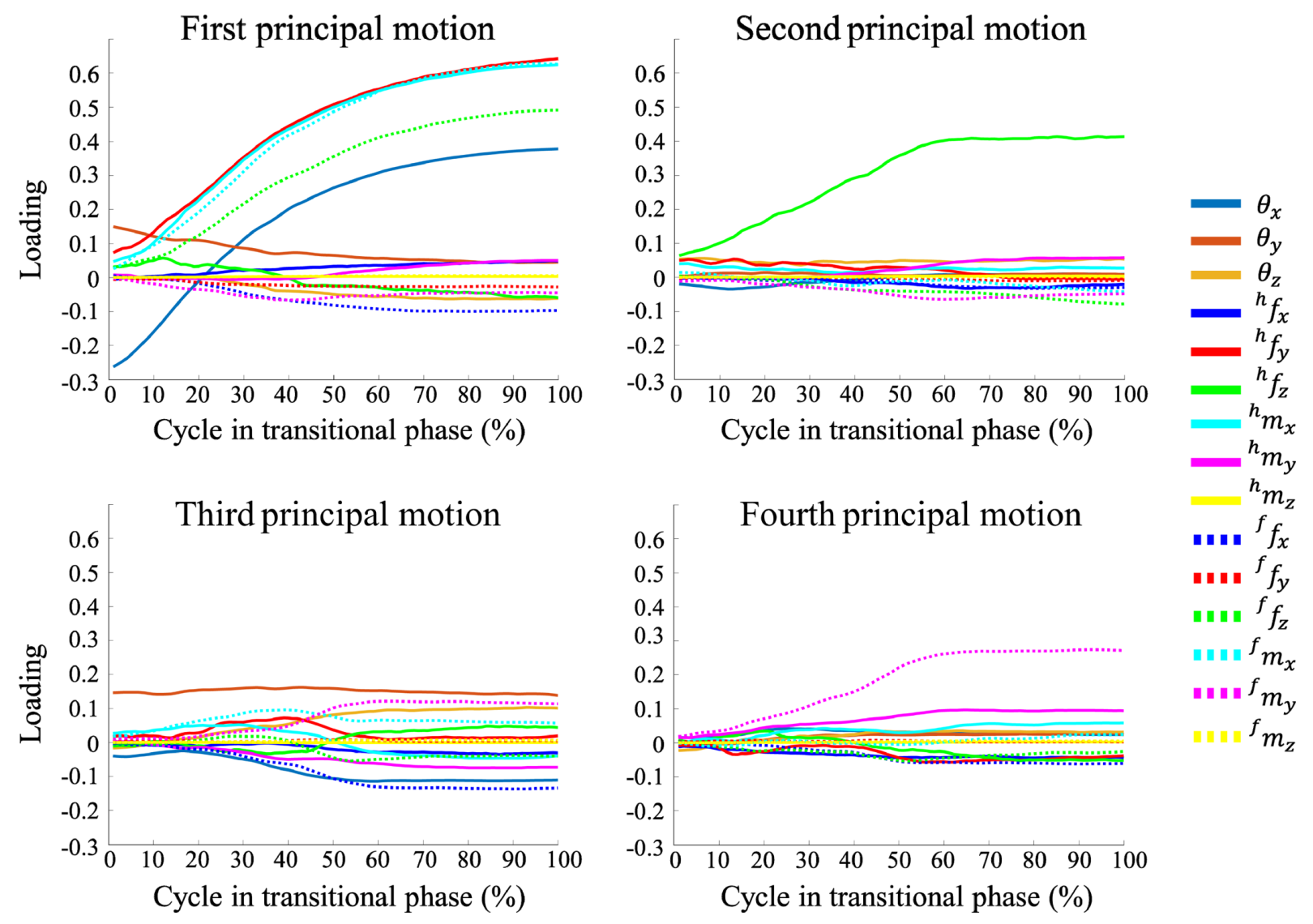

Fig. 2. First, second, third, and fourth principal motions.

each principal motion. In all the motions, the stretching techniques differed significantly among the physical therapists and patients. Furthermore, in the first and third principal motions, an interaction between the two factors existed.

\section{DISCUSSION}

While several studies on the methods and effects of stretching have been presented, manual stretching techniques have rarely been analyzed. In this study, we examined the manual foot stretching techniques used by multiple physical therapists for multiple patients and analyzed them statistically by performing a principal motion analysis to evaluate the differences among the techniques used by physical therapists.

Currently, the statistical methods used to analyze multidimensional time-series data of multiple trials such as stretching studies have not been established. The principal motion analysis is primarily used to generate motion in the engineering field and has become a major method in recent years ${ }^{9-11,13)}$. We used this method for the analysis of manual stretching. Thus, we successfully extracted the features of manual stretching. Moreover, by a two-way analysis of variance of the scores calculated from the principal motion analysis, we statistically determined whether a significant difference exists among the stretching techniques used by physical therapists. This enabled us to analyze manual stretching in detail quantitatively and statistically for the first time to our knowledge. The ability to analyze from a statistical perspective the stretching techniques that are applied empirically by therapists is a major advancement in rehabilitation, which requires evidence-based medicine. Furthermore, this analysis method can also be applied to the stretching of other body parts. Moreover, the method can be used to analyze multidimensional time-series data similar to stretching data such standing and walking motion data.

In this study, to identify the characteristics of the stretching techniques for spastic foot, we obtained the contributing motions to the stretching techniques, which in descending order are the motion to achieve dorsiflexion of the foot, the motion to push/pull the heel, the motion to invert/evert the entire foot, and the motion to invert/evert the forefoot. General foot dorsiflexion stretching relies on the stretching of the lower leg back muscles. The triceps surae muscles, which are large muscles on the surface, run in the direction of the long axis of the lower leg. Therefore, these muscles are stretched when a force is applied in the dorsiflexion direction. Moreover, because the triceps surae muscles are attached to the calcaneal bone, the stretching force is accurately applied to the triceps on pulling the calcaneal bone. Hence, the characteristics of the stretch- 


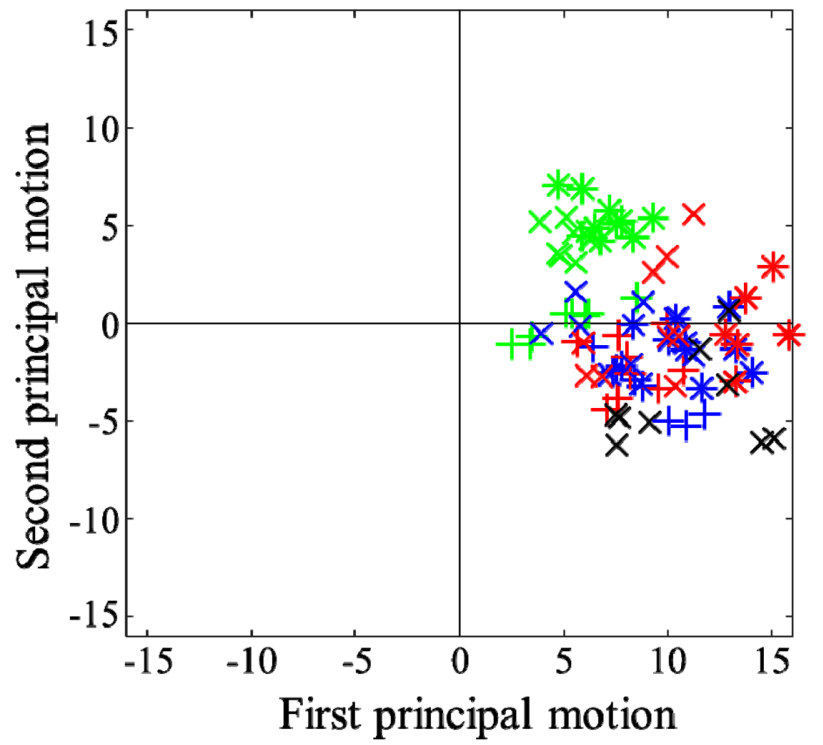

$\begin{array}{cc}+\mathrm{ptA} & \bullet \mathrm{PT} 1 \\ \text { * } \mathrm{ptB} & \mathrm{PT} 2 \\ \times \mathrm{ptC} & \bullet \mathrm{PT} 3 \\ & \bullet \mathrm{PT} 4\end{array}$

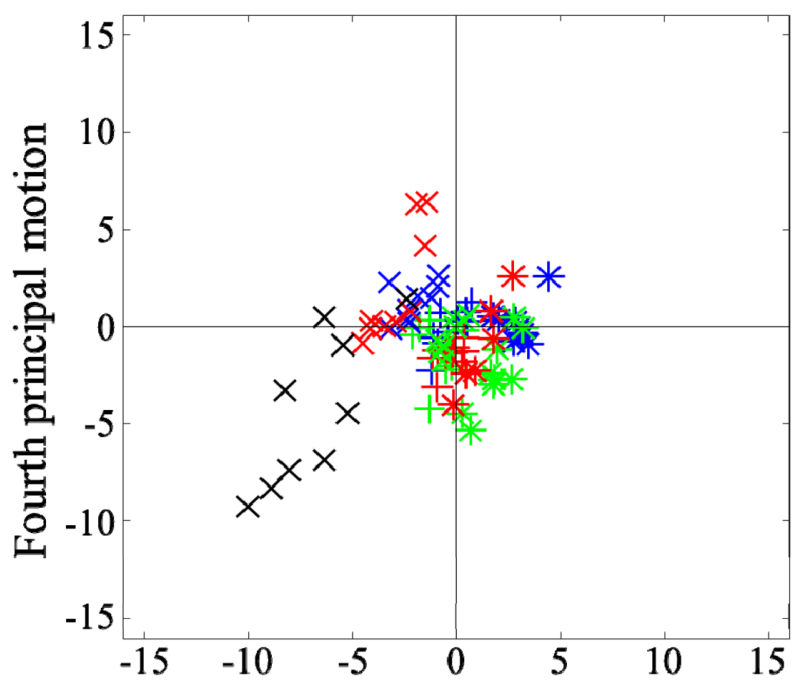

$\left[\begin{array}{c}+ \text { ptA-PT1 } \\ + \text { ptA-PT2 } \\ + \text { ptA-PT3 } \\ * \text { ptB-PT1 } \\ * \text { ptB-PT2 } \\ * \text { ptB-PT3 } \\ \times \text { ptC-PT1 } \\ \times \text { ptC-PT2 } \\ \times \text { ptC-PT3 } \\ \times \text { ptC-PT4 }\end{array}\right]$

PT : Physical therapist

pt : Patient

Third principal motion

Fig. 3. Scores of principal motions. Comparison between the first and second principal motions, and between the third and fourth principal motions.

Table 1. Scores of the patient-and-physical therapist pairs and results of the two-way analysis of variance

\begin{tabular}{|c|c|c|c|c|c|c|c|c|c|c|c|c|c|c|c|}
\hline \multirow{3}{*}{$\begin{array}{l}\text { Prin- } \\
\text { cipal } \\
\text { motion }\end{array}$} & \multicolumn{3}{|c|}{ Patient A } & \multicolumn{2}{|c|}{ Patient B } & \multicolumn{3}{|c|}{ Patient C } & \multirow{3}{*}{ PT 3} & \multicolumn{6}{|c|}{ Two-way ANOVA } \\
\hline & \multirow[t]{2}{*}{ PT 1} & \multirow[t]{2}{*}{ PT 2} & \multirow[t]{2}{*}{ PT 3} & \multirow[t]{2}{*}{ PT 1} & \multirow[t]{2}{*}{ PT 2} & \multirow[t]{2}{*}{ PT 3} & \multirow[t]{2}{*}{ PT 1} & \multirow[t]{2}{*}{ PT 2} & & \multicolumn{2}{|c|}{$\begin{array}{l}\text { Main effect } \\
\text { PT }\end{array}$} & \multicolumn{2}{|c|}{$\begin{array}{c}\text { Main effect } \\
\text { Patient }\end{array}$} & \multicolumn{2}{|c|}{ Interaction } \\
\hline & & & & & & & & & & $\mathrm{F}$ & p-value & $\mathrm{F}$ & $\mathrm{p}$-value & $\mathrm{F}$ & p-value \\
\hline 1 st & $\begin{array}{c}8.8 \pm \\
1.8\end{array}$ & $\begin{array}{l}5.2 \pm \\
1.8\end{array}$ & $\begin{array}{c}8.3 \pm \\
1.6\end{array}$ & $\begin{array}{c}11.4 \pm \\
1.8\end{array}$ & $\begin{array}{c}7.1 \pm \\
1.4\end{array}$ & $\begin{array}{c}14.1 \pm \\
1.2\end{array}$ & $\begin{array}{c}7.8 \pm \\
2.4\end{array}$ & $\begin{array}{c}5.4 \pm \\
0.9\end{array}$ & $\begin{array}{c}8.9 \pm \\
2.0\end{array}$ & 52.2 & $<0.001$ & 37.5 & $<0.001$ & 4.2 & $<0.005$ \\
\hline 2nd & $\begin{array}{c}-3.3 \pm \\
1.4\end{array}$ & $\begin{array}{c}0.5 \pm \\
1.7\end{array}$ & $\begin{array}{l}-2.2 \pm \\
1.5\end{array}$ & $\begin{array}{c}-1.0 \pm \\
1.3\end{array}$ & $\begin{array}{c}5.4 \pm \\
1.0\end{array}$ & $\begin{array}{c}-0.4 \pm \\
1.9\end{array}$ & $\begin{array}{l}-0.8 \pm \\
1.7\end{array}$ & $\begin{array}{c}4.4 \pm \\
0.8\end{array}$ & $\begin{array}{c}0.1 \pm \\
3.1\end{array}$ & 69.7 & $<0.001$ & 26.1 & $<0.001$ & 2.1 & 0.089 \\
\hline $3 \mathrm{rd}$ & $\begin{array}{c}-0.3 \pm \\
0.8\end{array}$ & $\begin{array}{c}-0.8 \pm \\
0.8\end{array}$ & $\begin{array}{c}-0.3 \pm \\
0.6\end{array}$ & $\begin{array}{c}3.0 \pm \\
0.8\end{array}$ & $\begin{array}{c}1.9 \pm \\
0.9\end{array}$ & $\begin{array}{c}1.1 \pm \\
1.0\end{array}$ & $\begin{array}{c}-2.0 \pm \\
0.9\end{array}$ & $\begin{array}{c}-0.3 \pm \\
0.6\end{array}$ & $\begin{array}{c}-2.9 \pm \\
1.2\end{array}$ & 10.9 & $<0.001$ & 130.6 & $<0.001$ & 11.1 & $<0.001$ \\
\hline 4 th & $\begin{array}{c}-0.1 \pm \\
1.1\end{array}$ & $\begin{array}{c}-1.2 \pm \\
1.4\end{array}$ & $\begin{array}{c}-1.5 \pm \\
0.7 \\
\end{array}$ & $\begin{array}{c}0.1 \pm \\
1.0\end{array}$ & $\begin{array}{c}-2.4 \pm \\
1.9\end{array}$ & $\begin{array}{c}-1.3 \pm \\
2.3\end{array}$ & $\begin{array}{c}1.3 \pm \\
1.0\end{array}$ & $\begin{array}{c}-0.5 \pm \\
0.8\end{array}$ & $\begin{array}{c}1.9 \pm \\
2.9\end{array}$ & 8.5 & $<0.001$ & 13.3 & $<0.001$ & 2.1 & 0.096 \\
\hline
\end{tabular}

Mean \pm standard deviation

PT: Physical therapist. 
ing techniques extracted as the first and second principal motions are considered appropriate. On the other hand, the third and fourth principal motions were techniques to apply force in the direction of the rotation. The participants in this study were post-stroke patients, whose posterior tibial muscles, in addition to the triceps surae muscles, were hypertonic. Therefore, the foot may have been deformed in the inversion position. The posterior tibial muscle acts on the plantar flexion and inversion of the foot ${ }^{14)}$. The third and fourth principal motions could have been produced because physical therapists stretch the shortened muscles while feeling the tension of muscles related to foot rotation. This suggests that the physical therapist applies manual stretching adaptively in accordance with the symptoms exhibited by the patient.

On the other hand, according to the results of the two-way analysis of variance, in all the motions, the stretching techniques significantly varied among physical therapists or patients. Naturally, the stretching procedures differ between patients according to the degree of tonus in the target muscle in individual patients. This indicates that the condition of the patient determines the stretching procedure.

The fact that the stretching techniques differ significantly among the physical therapists implies that the procedures differ even for the same patient. Moreover, in the first and third principal motions, an interaction between the two factors, the physical therapist and the patient, was observed. This implies that differences in these motions occurred only for a particular combination of therapists and patients. We predicted that this difference is directly related to the difference in treatment effectiveness. A detailed examination should be conducted in the future to clarify under which conditions this difference arises and which procedure is effective.

The limitation of this study is that only few physical therapists participated in the experiment. If data from more physical therapists are acquired, the results may differ. For example, we can investigate how the difference in years of experience among the therapists affects the results. Further testing of more participants is also desirable. In a future work, the footstretching technique used by each physical therapist and its effectiveness for the patient should be analyzed simultaneously to establish an effective stretching method. Moreover, several previous studies developed automatic machines for ankle stretching ${ }^{15-18)}$. Although currently, no such machine has been commercialized, the analysis of foot-stretching techniques in this study will help in their development.

The manual stretching techniques used by physical therapists for the ankle joints were statistically analyzed using an instrumented cuff and a motion-capturing system and the principal motion analysis. As a result, the principal motions were successfully extracted that indicated the average stretching techniques of the four physical therapists and several characteristic techniques. Furthermore, for each principal motion, the stretching procedures were significantly different among the physical therapists and patients. These analysis methods will help quantitatively and statistically study the stretching techniques used by physical therapists.

\section{Funding}

This work was supported by JSPS KAKENHI Grant No. JP17K13108 and JP20K19403 and a Grant-in-Aid for individual research in Aichi Medical College.

\section{Conflict of interest}

There are no conflicts of interest to declare.

\section{REFERENCES}

1) Medeiros DM, Martini TF: Chronic effect of different types of stretching on ankle dorsiflexion range of motion: systematic review and meta-analysis. Foot, 2018, 34: 28-35. [Medline] [CrossRef]

2) Lima BN, Lucareli PR, Gomes WA, et al.: The acute effects of unilateral ankle plantar flexors static-stretching on postural sway and gastrocnemius muscle activity during single-leg balance tasks. J Sports Sci Med, 2014, 13: 564-570. [Medline]

3) Young R, Nix S, Wholohan A, et al.: Interventions for increasing ankle joint dorsiflexion: a systematic review and meta-analysis. J Foot Ankle Res, 2013, 6 : 46. [Medline] [CrossRef]

4) Macklin K, Healy A, Chockalingam N: The effect of calf muscle stretching exercises on ankle joint dorsiflexion and dynamic foot pressures, force and related temporal parameters. Foot, 2012, 22: 10-17. [Medline] [CrossRef]

5) Hirata K, Kanehisa H, Miyamoto N: Acute effect of static stretching on passive stiffness of the human gastrocnemius fascicle measured by ultrasound shear wave elastography. Eur J Appl Physiol, 2017, 117: 493-499. [Medline] [CrossRef]

6) Maeda N, Urabe Y, Tsutsumi S, et al.: The acute effects of static and cyclic stretching on muscle stiffness and hardness of medial gastrocnemius muscle. J Sports Sci Med, 2017, 16: 514-520. [Medline]

7) Nakamura M, Ikezoe T, Nishishita S, et al.: Acute effects of static stretching on the shear elastic moduli of the medial and lateral gastrocnemius muscles in young and elderly women. Musculoskelet Sci Pract, 2017, 32: 98-103. [Medline] [CrossRef]

8) Morse CI, Degens H, Seynnes OR, et al.: The acute effect of stretching on the passive stiffness of the human gastrocnemius muscle tendon unit. J Physiol, 2008, 586: 97-106. [Medline] [CrossRef]

9) Park FC, Jo K: Movement primitives and principal component analysis. In: On advances in robot kinematics. Dordrecht: Springer, 2004, pp 421-430.

10) Nakanishi H, Kanata S, Hattori H, et al.: Extraction of coordinative structures of motions by segmentation using singular spectrum transformation. J Adv Comput Intell Intell Inf, 2011, 15: 1019-1029. [CrossRef] 
11) Morishima S, Ayusawa K, Yoshida E, et al.: Whole-body motion retargeting using constrained smoothing and functional principle component analysis. IEEE International Conference on Humanoid Robots, 2016, 294-299.

12) Yamada N, Okamoto S, Okumura H, et al.: Similarities and differences in manual stretching of physical therapists for equinovarus. IEEE/SICE International Symposium on System Integration, 2014, 490-495.

13) Lim B, Ra S, Park FC: Movement primitives, principal component analysis, and the efficient generation of natural motions. Proceedings of the 2005 IEEE International Conference on Robotics and Automation, 2005, 4630-4635.

14) Tortora GJ, Nielsen MT: Principles of human anatomy, 11th edition. Hoboken: John Wiley \& Sons, Inc., 2009.

15) Shiraishi Y, Okamoto S, Yamada N, et al.: Pneumatically-driven stretching machine for ankle dorsiñexion: safety concepts and effectiveness test involving healthy young subjects. Robomech J, 2020, 7: 10. [CrossRef]

16) Zhou Z, Sun Y, Wang N, et al.: Robot-assisted rehabilitation of ankle plantar flexors spasticity: a 3-month study with proprioceptive neuromuscular facilitation. Front Neurorobot, 2016, 10: 16. [Medline] [CrossRef]

17) Yamada N, Okamoto S, Akiyama Y, et al.: Ankle stretching rehabilitation machine for equinovarus: design and evaluation from clinical aspects. IEEE International Conference on Systems, Man, and Cybernetics, 2017, 1687-1692.

18) Kimura T, Okamoto S, Yamada N, et al.: Ankle stretching rehabilitation machine for equinovarus: automation of eversion and flexion control. IEEE International Conference on Systems, Man, and Cybernetics, 2017, 2696-2700. 